\title{
A simple algorithm for constructing Szemerédi's Regularity Partition
}

\author{
Alan Frieze \\ Department of Mathematical Sciences, \\ Carnegie Mellon University. \\ alan@random.math.cmu.edu* \\ Ravi Kannan \\ Department of Computer Science, \\ Yale University. \\ kannan@cs . yale.edu ${ }^{\dagger}$
}

Submitted: November 25, 1998; Accepted: March 15, 1999.

\begin{abstract}
We give a simple constructive version of Szemerédi's Regularity Lemma, based on the computation of singular values of matrices. Mathematical Reviews Subject Numbers: 05C85, 68R10.
\end{abstract}

\section{Introduction}

"Szemerédi's Regularity Lemma [9] is one of the most powerful tools of (extremal) graph theory". One can only agree with that opening sentence of the

*Supported in part by NSF grant CCR-9530974.

${ }^{\dagger}$ Supported in part by NSF grant CCR-9528973. 
paper by Komlós and Simonovits [6]. It has many, many applications and we refer the reader to this excellent survey.

The Regularity lemma is often used to prove the existence of certain objects and if in addition one wants to construct them, then one needs a constructive version of the lemma. This was provided by Alon, Duke, Lefmann, Rödl and Yuster [1]. Subsequently, Frieze and Kannan [3, 4] gave a different version and extended it to deal with hypergraphs, (see also Czygrinow and Rödl [2]).

In this note, we give another construction based on the construction of singular values of matrices. The proofs in $[1,3,4]$ are somewhat technical. The result of this paper follows quite easily from a simple lemma relating non-regularity and largeness of singular values.

\subsection{Szemerédi's Lemma}

Let $G=(V, E)$ be a graph with $n$ vertices and let $\mathbf{A}$ be its adjacency matrix. For a disjoint pair of subsets $A, B \subseteq V$ let $e(A, B)$ denote the number of edges between $A$ and $B$. The density $d(A, B)$ is defined by

$$
d(A, B)=\frac{e(A, B)}{|A||B|} .
$$

A disjoint pair $A, B \subseteq V$ is said to be $\epsilon-$ regular if for every $X \subseteq A$ with $|X| \geq \epsilon|A|$ and $Y \subseteq B$ with $|Y| \geq \epsilon|B|$, we have

$$
|d(X, Y)-d(A, B)| \leq \epsilon
$$

Theorem 1 (Szemerédi's Regularity Lemma) For every $\epsilon>0$ and integer $m>0$ there are integers $P(\epsilon, m), Q(\epsilon, m)$ with the following property: for every graph $G=(V, E)$ with $n \geq P(\epsilon, m)$ vertices there is a partition $\mathcal{P}$ of $V$ into $k+1$ classes $V_{0}, V_{1}, \ldots, V_{k}$ such that

- $m \leq k \leq Q(\epsilon, m)$.

- $\left|V_{1}\right|=\left|V_{2}\right|=\cdots=\left|V_{k}\right|$.

- All but at most $\epsilon k^{2}$ of the pairs $\left(V_{i}, V_{j}\right)$ are $\epsilon$-regular.

- $\left|V_{0}\right| \leq \epsilon n$. 
A partition satisfying the second criterion is called equitable. $V_{0}$ is called the exceptional class.

Following [9], for every equitable partition $\mathcal{P}$ into $k+1$ classes we define a number called the index of $\mathcal{P}$.

$$
\operatorname{ind}(\mathcal{P})=\frac{1}{k^{2}} \sum_{1 \leq r, s \leq k} d\left(V_{i}, V_{j}\right)^{2} .
$$

A crucial lemma proved in [9] and stated in the following form in [1] states:

Lemma 1 Fix $k$ and $\gamma$ and let $G=(V, E)$ be a graph with $n$ vertices. Let $\mathcal{P}$ be an equitable partition of $V$ into classes $V_{0}, V_{1}, \ldots, V_{k}$. Assume $\left|V_{1}\right|>$ $4^{2 k}$ and $4^{k}>600 \gamma^{-2}$. Given proofs that more than $\gamma k^{2}$ pairs $\left(V_{r}, V_{s}\right)$ are not $\gamma$-regular (where by proofs we mean subsets $X=X(r, s) \subseteq V_{r}, Y=$ $Y(r, s)=\subseteq V_{s}$ that violate the $\gamma$-regularity of $\left.\left(V_{r}, V_{s}\right)\right)$ we can find in $O(n)$ time an equitable partition $\mathcal{P}^{\prime}$ (which is a refinement of $\mathcal{P}$ ) into $1+k 4^{k}$ classes, with an exceptional class of cardinality at most

$$
\left|V_{0}\right|+n / 4^{k}
$$

and such that

$$
\operatorname{ind}\left(\mathcal{P}^{\prime}\right) \geq \operatorname{ind}(\mathcal{P})+\gamma^{5} / 20
$$

We first describe a procedure for finding a proof that a pair is not $\gamma$-regular; this will be the central part. Then we complete the algorithm with this procedure on hand using the above lemma.

\subsection{Singular Values}

An $m \times n$ matrix $\mathbf{A}$ has a Singular Value Decomposition into the sum of rank one matrices, see for example Golub and Van Loan [5]. It has many important applications. The first singular value $\sigma_{1}$ is defined as

$$
\sigma_{1}(\mathbf{A})=\max _{|x|=|y|=1}\left|x^{T} \mathbf{A} y\right|
$$

This value can be computed with high accuracy in polynomial time [5]. It is the square root of the largest eigenvalue of $\mathbf{A}^{T} \mathbf{A}$.

For the following lemma, $\mathbf{W}$ is a $p \times q$ matrix with rows indexed by $R$, columns indexed by $C$. We assume that $\|\mathbf{W}\|_{\infty}=\max _{i \in R, j \in C}|\mathbf{W}(i, j)| \leq 1$. 
For $S \subseteq R, U \subseteq C$ we let

$$
\mathbf{W}(S, T)=\sum_{i \in S} \sum_{j \in T} \mathbf{W}(i, j)=x_{S}^{T} \mathbf{W} x_{U}
$$

where $x_{S}$ is the $0-1$ indicator vector of $S$ i.e. $\left(x_{S}\right)_{i}=1$ iff $i \in S$.

Let $\mathbf{A}$ be the adjacency matrix of $G$. Suppose we now have a partition of $V$ into $V_{1}, V_{2}, \ldots$ and we wish to check whether $\left(V_{r}, V_{s}\right)$ form a $\gamma$-regular pair for some $\gamma$. We let $R=V_{r}, C=V_{s}$ and let $\mathbf{A}_{r, s}$ be the $R \times C$ submatrix of A corresponding to these rows and columns. Let

$$
d=\frac{1}{\left|V_{r}\right|\left|V_{s}\right|} \sum_{i \in V_{r}} \sum_{j \in V_{s}} \mathbf{A}(i, j)
$$

be the average of the entries in $A_{r, s}$. Let $\mathbf{D}$ be the $R \times C$ matrix with all entries equal to $d$. Let $\mathbf{W}=\mathbf{W}_{r, s}=\mathbf{A}_{r, s}-\mathbf{D}$. Re-phrasing the definition of a regular pair we see that

$$
\left(V_{r}, V_{s}\right) \text { is an } \epsilon \text {-regular pair iff }|\mathbf{W}(S, T)| \leq \epsilon|S||T|
$$

for all $S \subseteq R,|S| \geq \epsilon|R|, T \subseteq C,|T| \geq \epsilon|C|$.

The following lemma relates this all to $\sigma_{1}(\mathbf{W})$.

Lemma 2 Let $\mathbf{W}$ be an $R \times C$ matrix with $|R|=p,|C|=q$ and $\|\mathbf{W}\|_{\infty} \leq 1$ and $\gamma$ be a positive real.

(a) If there exist $S \subseteq R, T \subseteq C$ such that $|S| \geq \gamma p,|T| \geq \gamma q$ and $|\mathbf{W}(S, T)| \geq$ $\gamma|S||T|$ then $\sigma_{1}(\mathbf{W}) \geq \gamma^{3} \sqrt{p q}$.

(b) If $\sigma_{1}(\mathbf{W}) \geq \gamma \sqrt{p q}$ then there exist $S \subseteq R, T \subseteq C$ such that $|S| \geq$ $\gamma^{\prime} p,|T| \geq \gamma^{\prime} q$ and $|\mathbf{W}(S, T)| \geq \gamma^{\prime}|S||T|$ where $\gamma^{\prime}=\frac{\gamma^{3}}{108}$. Furthermore $S, T$ can be constructed in polynomial time.

\section{Proof}

(a) From (1) we see that

$$
\left|x_{S}^{T} \mathbf{W} x_{T}\right| \geq \gamma|S||T| \geq \gamma^{3} p q .
$$

Now let $\xi_{S}=x_{S} /\left|x_{S}\right|$ and $\xi_{T}=x_{T} /\left|x_{T}\right|$. Then

$$
\left|\xi_{S}^{T} \mathbf{W} \xi_{T}\right| \geq \gamma^{3} p q /\left(\left|\xi_{S}\right|\left|\xi_{T}\right|\right) \geq \gamma^{3} \sqrt{p q}
$$


since $\left|x_{S}\right| \leq \sqrt{p}$ and $\left|x_{T}\right| \leq \sqrt{q}$. This proves (a).

(b) W.l.o.g. we can choose $x, y$ such that $x^{T} \mathbf{W} y \geq \gamma \sqrt{p q},|x|=1,|y|=1$. Let $\beta>0(\beta$ will be later set to $3 / \gamma)$ and define $\hat{x}$ by

$$
\hat{x}_{i}= \begin{cases}x_{i}: & \text { if }\left|x_{i}\right| \leq \frac{\beta}{\sqrt{p}} \\ 0: & \text { otherwise }\end{cases}
$$

and define $\hat{y}$ by a similar truncation at $\beta / \sqrt{q}$.

Since $|x|=1$ we see that $I=\left\{i:\left|x_{i}\right| \geq \beta / \sqrt{p}\right\}$ has cardinality at most $p / \beta^{2}$. Let $\mathbf{W}_{1}$ be obtained from $\mathbf{W}$ by replacing elements in rows other than $I$ by zero. Then (using the standard inequality that for any vector $a$ and matrix $\mathbf{M}$, we have $\left|a^{T} \mathbf{M}\right| \leq\left.|a||| \mathbf{M}\right|_{F}$, where $\|\left.\mathbf{M}\right|_{F} ^{2}$ is the sum of squares of the entries of $\mathbf{M}$ )

$$
\left|(x-\hat{x})^{T} \mathbf{W} y\right|=\left|(x-\hat{x})^{T} \mathbf{W}_{1} y\right| \leq|x-\hat{x}||| \mathbf{W}_{1}\left\|_{F}|y| \leq\right\| \mathbf{W}_{1} \|_{F} \leq \frac{\sqrt{p q}}{\beta} .
$$

By a similar argument we obtain $\left|\hat{x}^{T} \mathbf{W}(y-\hat{y})\right| \leq \sqrt{p q} / \beta$. Thus

$$
\hat{x}^{T} \mathbf{W} \hat{y}=x^{T} \mathbf{W} y-(x-\hat{x})^{T} \mathbf{W} y-\hat{x}^{T} \mathbf{W}(y-\hat{y}) \geq(\gamma-2 / \beta) \sqrt{p q} .
$$

Let $\hat{\gamma}=\gamma-2 / \beta$. Then at least one of $\left(\hat{x}^{+}\right)^{T} \mathbf{W} \hat{y}^{+},\left(\hat{x}^{+}\right)^{T} \mathbf{W} \hat{y}^{-},\left(\hat{x}^{-}\right)^{T} \mathbf{W} \hat{y}^{+}$, $\left(\hat{x}^{-}\right)^{T} \mathbf{W} \hat{y}^{-}$is at least $\hat{\gamma} \sqrt{p q} / 4$. Here $\xi^{+}$is obtained from $\xi \in \boldsymbol{R}^{p}$ by putting $\xi_{i}^{+}=\max \left\{0, \xi_{i}\right\} \cdot \xi^{-}=-\left((-\xi)^{+}\right)$.

Suppose without loss of generality that $\left(\hat{x}^{+}\right)^{T} \mathbf{W} \hat{y}^{-} \geq \hat{\gamma} \sqrt{p q} / 4$. (The proof for the other cases is similar.) We define random subsets $S, T$ as follows:

For each $i \in R$, put $i$ in $S$ with probability $\hat{x}_{i}^{+} \sqrt{p} / \beta$.

For each $j \in C$, put $j$ in $T$ with probability $-\hat{y}_{j}^{-} \sqrt{q} / \beta$.

Then

$$
\mathbf{E}(\mathbf{W}(S, T))=-\left(\hat{x}^{+}\right)^{T} \mathbf{W} \hat{y}^{-}\left(\sqrt{p q} / \beta^{2}\right) \leq-\hat{\gamma} p q /\left(4 \beta^{2}\right) .
$$

Thus there exist $S, T$ such that $\mathbf{W}(S, T) \leq-\hat{\gamma} p q /\left(4 \beta^{2}\right)$. Furthermore, such $S, T$ can easily be constructed in $O(p q)$ time using the method of conditional expectations [7] and [8]. Indeed for any $r \in R$ we have

$\mathbf{E}(\mathbf{W}(S, T))=\mathbf{E}(\mathbf{W}(S, T) \mid r \in S) \operatorname{Pr}(r \in S)+\mathbf{E}(\mathbf{W}(S, T) \mid r \notin S) \operatorname{Pr}(r \notin S)$

and fixing $r \in S$ or $r \notin S$ essentially reduces the size of $R$ by one. 
Putting $\beta=3 / \gamma$ we get $|\mathbf{W}(S, T)| \geq \gamma^{3} p q / 108$. We need only verify that $S, T$ are not too small in order to complete the proof of (b). But this follows immediately from $|S||T| \geq|\mathbf{W}(S, T)|$.

We can combine Lemmas 1 and 2 to make an algorithm for finding an $\epsilon$ regular partition, much as in [1].

1. Arbitrarily divide the vertices of $G$ into an equitable partition $\mathcal{P}_{1}$ with classes $V_{0}, V_{1}, \ldots, V_{b}$ where $\left|V_{i}\right|=\lfloor n / b\rfloor$ and hence $\left|V_{0}\right|<b$. denote $k_{1}=b$.

2. For every pair $\left(V_{r}, V_{s}\right)$ of $\mathcal{P}_{i}$, compute $\sigma_{1}\left(\mathbf{W}_{r, s}\right)$. If the pair $\left(V_{r}, V_{s}\right)$ are not $\epsilon$-regular then by Lemma 2 we obtain a proof that they are not $\gamma=\epsilon^{9} / 108$-regular.

3. If there are at most $\epsilon\left(\begin{array}{c}k_{i} \\ 2\end{array}\right)$ pairs that produce proofs of non $\gamma$-regularity then halt. $\mathcal{P}_{i}$ is $\epsilon$-regular.

4. Apply Lemma 1 where $\mathcal{P}=\mathcal{P}_{i}, k=k_{i}, \gamma=\epsilon^{9} / 108$ and obtain a partition $\mathcal{P}^{\prime}$ with $1+k_{i} 4^{k_{i}}$ classes.

5. Let $k_{i+1}=k_{i} 4^{k_{i}}, \mathcal{P}_{i+1}=\mathcal{P}^{\prime}, i=i+1$ and go to Step 2 .

The algorithm finishes in at most $O\left(\epsilon^{-45}\right)$ steps with an $\epsilon$-regular partition, since ind $\leq 1 / 2$ and each non-terminating step increases the index by $\gamma^{5} / 20=$ $\Omega\left(\epsilon^{45}\right)$.

\section{References}

[1] N.Alon, R.A.Duke, H.Lefmann, V.Rödl and R.Yuster, The algorithmic aspects of the Regularity Lemma, Journal of Algorithms 16 (1994) 80109.

[2] A.Czygrinow and V.Rödl, Algorithmic Regularity Lemma for Hypergraphs, in preparation.

[3] A.M.Frieze and R.Kannan, The Regularity Lemma and approximation schemes for dense problems, Proceedings of the 37th Annual IEEE Symposium on Foundations of Computing, (1996) 12-20. 
[4] A.M.Frieze and R.Kannan, Quick approximations to matrices and applications, to appear in Combinatorica.

[5] G.H.Golub and C.F.Van Loan, Matrix Computations, Johns Hopkins University Press, London, 1989.

[6] J.Komlós and M.Simonovits, Szemerédi's regularity Lemma and its applications in graph theory, Combinatorics, Paul Erdős is Eighty, Bolyai Society Mathematical Studies, 2, D.Miklós, V.T.Sós and T.Szőnyi Eds., (1996) 295-352.

[7] P.Raghavan, Probabilistic construction of deterministic algorithms: Approxiamting packing integer programs, Journal of Computer and System Sciences 37 (1988) 130-143.

[8] J.Spencer, Ten lectures on the probabilistic method, SIAM, Philadelphia, 1987.

[9] E.Szemerédi, Regular partitions of graphs, Proceedings, Colloque Inter. CNRS (J.-C. Bermond, J.-C.Fournier, M.Las Vergnas and D.Sotteau, Eds.) (1978) 399-401. 